\title{
Cryopreserved potato shoot tips showed genotype-specific response to sucrose concentration in rewarming solution (RS)
}

\author{
Rainer Vollmer $^{1}\left[\right.$ D $\cdot$ Rosalva Villagaray $^{1} \cdot$ Mario Castro ${ }^{1} \cdot$ Noelle L. Anglin $^{1} \cdot$ David Ellis $^{1}$
}

Received: 20 May 2018 / Accepted: 1 November 2018 / Published online: 10 November 2018

(c) The Author(s) 2018

\begin{abstract}
Cryopreservation protocols have been successfully developed for hundreds of species and thousands of genotypes in laboratories around the world. In many of the protocols, the rewarming process occurs in a rewarming solution (RS) with a high concentration of sucrose (0.8-1.2 M). Warming rate and associated conditions influence cell rehydration, loss of accumulated solutes (e.g. cryoprotectants) and recrystallization of small ice crystals in the nuclei. The need for, and effect of, high sucrose concentrations in the RS post-thawing regrowth rate after liquid nitrogen exposure was assessed in the range of 0.0-1.2 M sucrose with a set of 16 potato landraces cryopreserved with the PVS2-droplet vitrification method. The results showed no significant difference for the average recovery rate (81-87\%) between sucrose concentrations of $0.3 \mathrm{M}$ to $1.2 \mathrm{M}$. Fourteen of 16 accessions had their highest recovery rate with sucrose concentrations between 0.3 and $0.9 \mathrm{M}$. The experimental results were subsequently extended to a genetically diverse set of 85 potato accessions (nine taxa), which demonstrated significantly higher recovery rates of 55-61\% with RS sucrose concentrations of $0.3-0.9 \mathrm{M}$, compared to the sucrose concentrations of $0.0 \mathrm{M}(37 \%)$ and $1.2 \mathrm{M}(44 \%)$. Only one of 85 accessions showed its highest recovery rate with the routinely used RS sucrose concentration of $1.2 \mathrm{M}$. Of all the concentrations tested, $0.6 \mathrm{M}$ sucrose appeared to be the best bet in terms of recovery rates across the genotypes; therefore, our routine protocol has been changed from $1.2 \mathrm{M}$ sucrose to $0.6 \mathrm{M}$. The specific response to low $(0.0 \mathrm{M})$ and high RS sucrose concentrations $(1.2 \mathrm{M})$ was highly variable within species/subspecies and appears to be genotype specific. Thus, caution should be taken in generalizing experimental cryopreservation results obtained with a limited number of accessions to larger germplasm collections.
\end{abstract}

Keywords Potato $\cdot$ Solanum $\cdot$ Cryopreservation $\cdot$ Sucrose $\cdot$ Rewarming

\section{Introduction}

Sucrose-rich rewarming solutions (RS) are widely used for reducing osmotic shock during rapid rewarming of cryopreserved plant tissues and organs, or for diluting out harmful cryoprotectants after rewarming in warm water baths. The warming rate and its associated conditions influence cell rehydration, loss of accumulated solutes (e.g. cryoprotectants), and recrystallization of small ice crystals in the nuclei (Farrant et al. 1977; Brockbank et al. 2004). Potato shoot tips have been successfully cryopreserved with different cryopreservation methods (Schäfer-Menuhr et al. 1997; Hirai and

Communicated by Bart Panis.

Rainer Vollmer

r.vollmer@cgiar.org

1 Centro Internacional de la Papa, Lima, Peru
Sakai 1999; Yamamoto et al. 2012; Vollmer et al. 2016), but little information is available regarding the effect of the RS sucrose concentration on the recovery rate of cryopreserved potato shoot tips.

The International Potato Center (CIP) holds one of the largest and most diverse potato cryobanks worldwide, with a collection of 2330 potato landraces or $\sim 50 \%$ of the in trust potato germplasm collection currently preserved in liquid nitrogen. One of CIP's research priorities is to assess different variables of the cryopreservation process on a wide range of genotypes. The objective of the present study was to evaluate the effect of five different sucrose concentrations in the rewarming solution (RS) on the recovery rate of 16 potato landraces cryopreserved with the PVS2-droplet vitrification method, and then assess the results with a genetically diverse set of 85 potato accessions, including $82 \%$ of CIP's mini-core potato collection (http://genebank.cipotato. org/gringlobal/methodaccession.aspx). 
The accessions used in this study belong to nine different taxa and varying ploidy levels from CIP's in vitro potato collection (relative size of taxa in the in vitro genebank is indicated in parenthesis): $S$. tuberosum subsp. andigenum ( $67.1 \%)$, S. stenotomum subsp. stenotomum ( 5.8\%), S. phureja ( 4.3\%), S. tuberosum subsp. tuberosum ( 3.4\%), S. $\times$ chaucha $(\sim 2.6 \%)$, S. stenotomum subsp. goniocalyx ( 2.1\%), S. × juzepczukii ( 0.6\%), S. × ajanhuiri $(\sim 0.3 \%)$ and $S . \times$ curtilobum $(\sim 0.2 \%)$ (taxonomy based on Hawkes 1990).

\section{Materials and methods}

\section{Propagation of plant material}

In vitro potato plantlets were subcultured in $25 \times 150 \mathrm{~mm}$ test tubes every 3-4 weeks onto modified Murashige and Skoog medium (MS), with 5-6 plants per tube. The medium contained full MS salts and the standard MS organic compounds (Murashige and Skoog 1962) supplemented with $25 \mathrm{~g} \mathrm{~L}^{-1}$ of sucrose and $3.0 \mathrm{~g} \mathrm{~L}^{-1}$ of Phytagel ${ }^{\mathrm{TM}}$. The $\mathrm{pH}$ was adjusted to $5.60 \pm 0.02$ prior to autoclaving. Plants were incubated at $18-22{ }^{\circ} \mathrm{C}$ with a $16 / 8 \mathrm{~h}$ photoperiod and light intensity of $80-100 \mu \mathrm{mol} \mathrm{m}^{-2} \mathrm{~s}^{-1}$. Terminal buds were subcultured in deep petri dishes (70 buds per petri dish) for the final transfer and incubated for 3 weeks at $6-8{ }^{\circ} \mathrm{C}$ at low light intensity (15-25 $\mu \mathrm{mol} \mathrm{m} \mathrm{m}^{-2} \mathrm{~s}^{-1}$ ), with a photoperiod of $16 \mathrm{~h} / 8 \mathrm{~h}$.

\section{Excision of shoot tips}

Shoot tips were excised from 3-week-old cold-acclimated in vitro plantlets. Excised shoot tips contained 3-4 leaf primordia (length: $0.8-1.2 \mathrm{~mm}$; width: $0.4-0.7 \mathrm{~mm}$ ). The excised shoot tips were placed successively onto sterile filter paper $(1 \times 1 \mathrm{~cm})$, until each filter paper contained five shoot tips. The filter papers were supported on modified MS medium supplemented with $0.04 \mathrm{mg} \mathrm{L}^{-1}$ kinetin, $0.1 \mathrm{mg} \mathrm{L}^{-1}$ gibberellic acid, $25 \mathrm{~g} \mathrm{~L}^{-1}$ sucrose and $2.8 \mathrm{~g} \mathrm{~L}^{-1}$ Phytagel ${ }^{\mathrm{TM}}$ $(\mathrm{pH} 5.60 \pm 0.02)$ in standard petri dishes.

\section{Cryoprotection}

The filter papers containing five shoot tips each were placed into sterile $8.0 \mathrm{~mL}$ screw cap test tubes with $2.0 \mathrm{~mL}$ filtersterilized Loading Solution (LS) [liquid MS medium, 2.0 M glycerol, $0.4 \mathrm{M}$ sucrose, $\mathrm{pH} 5.80 \pm 0.02]$. Shoot tips were treated at room temperature $\left(22-26^{\circ} \mathrm{C}\right)$ for $20 \mathrm{~min}$. Using a sterile glass Pasteur pipette with a $1.0 \mathrm{~mL}$ rubber bulb, LS was carefully removed and replaced with $2.0 \mathrm{~mL}$ of ice-cold filter-sterilized Plant Vitrification Solution 2 (PVS2) [liquid MS medium, 3.28 M glycerol, 2.42 M ethylene glycol, 1.9 M dimethylsulfoxide, $0.4 \mathrm{M}$ sucrose, $\mathrm{pH} 5.80 \pm 0.02]$. Shoot tips were treated with PVS2 on ice $\left(0{ }^{\circ} \mathrm{C}\right)$ for $50 \mathrm{~min}$ at ambient light conditions in the flow chamber.

\section{Cooling in liquid nitrogen (LN)}

Shoot tips were removed from the vial using a sterile glass Pasteur pipette ( $\varnothing$ of opening: $2.7-2.8 \mathrm{~mm}$ ), the pipette's tip was placed on the bottom of the test tube, the shoot tips plus the PVS solution were drawn up into the pipette and the PVS2 was expelled from the pipette until the five shoot tips were contained in approximately $10 \mu \mathrm{L}$ of PVS2 in the tip of the pipette. Shoot tips were placed along with the $10 \mu \mathrm{L}$ PVS2-droplet on a sterile aluminum foil strip $(5 \times 20 \mathrm{~mm})$ which was slightly folded $(2-3 \mathrm{~mm})$ at one end. The aluminum foil strip was quickly plunged into $\mathrm{LN}\left(-196{ }^{\circ} \mathrm{C}\right)$ and transferred under $\mathrm{LN}$ into a $1.8 \mathrm{~mL}$ cryovial.

\section{Rewarming}

After a minimum of $24 \mathrm{~h}$ in LN, each aluminum foil strip (with five shoot tips) was removed from the cryovial and plunged into a sterile $8.0 \mathrm{~mL}$ screw cap test tube containing $4.0 \mathrm{~mL}$ room temperature filter-sterilized RS (liquid MS medium, $0.0-1.2 \mathrm{M}$ of sucrose, $\mathrm{pH} 5.80 \pm 0.02$ ). RS was supplemented with five different sucrose concentrations: $0.0 \mathrm{M}$, $0.3 \mathrm{M}, 0.6 \mathrm{M}, 0.9 \mathrm{M}$, and $1.2 \mathrm{M}$ (control treatment). The RS sucrose concentration of $1.2 \mathrm{M}$ was routinely used at CIP for more than 10 years (Panta et al. 2015; Vollmer et al. 2017). Shoot tips were rewarmed in rewarming solution (RS) for $20 \mathrm{~min}$ at room temperature $\left(20-24^{\circ} \mathrm{C}\right)$ under ambient light conditions (20-30 $\mu \mathrm{mol} \mathrm{m}^{-2} \mathrm{~s}^{-1}$ ). Shoot tips were removed from the test tubes with a Pasteur pipette and placed for $60-120 \mathrm{~s}$ on a sterile filter paper $(1 \times 1 \mathrm{~cm})$ supported on sterile bond papers to wick away excess RS.

\section{Recovery}

Shoot tips were dispersed on a larger filter paper $(2.5 \times 2 \mathrm{~cm})$ in a Petri dish containing Potato Recovery Medium I (PRMI) (MS medium, $0.4 \mathrm{mg} \mathrm{L}^{-1}$ kinetin, $0.1 \mathrm{mg} \mathrm{L}^{-1}$ gibberellic acid, $20 \mathrm{~mL} \mathrm{~L}^{-1}$ coconut water [Sigma C5915], $102.6 \mathrm{~g} \mathrm{~L}^{-1}$ sucrose $[=0.3 \mathrm{M}], 2.8 \mathrm{~g} \mathrm{~L}^{-1}$ Phytagel $^{\mathrm{TM}}, \mathrm{pH}$ $5.60 \pm 0.02$ ). Three filter papers, each containing five shoot tips were placed in each Petri dish. Shoot tips were incubated on PRM-I for 3 days in darkness at $18-22{ }^{\circ} \mathrm{C}$ followed by culturing on Potato Recovery Medium II (PRM-II) and III (PRM-III), for 3 days on each media (in darkness at $18-22{ }^{\circ} \mathrm{C}$ ). PRM-II and PRM-III contained the same composition as PRM-I, but with lower sucrose levels of $68.4 \mathrm{~g} \mathrm{~L}^{-1}$ $[=0.2 \mathrm{M}]$ and $34.2 \mathrm{~g} \mathrm{~L}^{-1}[=0.1 \mathrm{M}]$, respectively.

During culturing on PRM-I to PRM-III, shoot tips were supported on filter papers and transferred together to 
avoid damage to the shoot tips. After 9 days in darkness, shoot tips were removed from the filter paper and placed directly onto the culture medium with the same composition as PRM-I, except with $25.0 \mathrm{~g} \mathrm{~L}^{-1}[=0.07 \mathrm{M}]$ of sucrose (PRM-IV), and incubated for 4 days under diffuse light at $18-22{ }^{\circ} \mathrm{C}$ with a $16 \mathrm{~h} / 8 \mathrm{~h}$ photoperiod followed by normal light conditions (80-100 $\mu \mathrm{mol} \mathrm{m} \mathrm{m}^{-2} \mathrm{~s}^{-1}$ ). After 30 days, surviving shoot tips were transferred individually onto fresh PRM-IV in $13 \times 100 \mathrm{~mm}$ test tubes. Recovered samples without root formation were cut slightly at the base before transferring.

\section{Viability assessment}

Viability was assessed 60 days after rewarming. A shoot tip was classified as recovered, if it had developed into a complete and morphologically normal looking in vitro plant (elongated stem, functional apex, leaves, and roots). Shoot tips that developed into deformed plants showing only leaf formation or had signs of hyper-hydration (vitrification) were classified as survived, but not recovered.

\section{Data analysis}

Survival and recovery data were expressed as a percentage of the total assayed shoot tips. The experiment with the 16 potato accessions was repeated three times with sample sizes of $n=15$ and $n=10$ shoot tips per genotype for cooled $(+\mathrm{LN})$, and control samples $(-\mathrm{LN})$, respectively. The screening experiment (85 accessions) was repeated only one time, the three independent petri dishes $(n=5)$ assigned to each treatment of each accession were used for statistical analysis. The Anderson-Darling normality test showed p-values of $<0.005$ for the survival (SR) and recovery rates (RR). Medians were analyzed with the Kruskal-Wallis (K-W) multiple comparison test $(\mathrm{p}<0.05)$, a nonparametric test applicable to small sample sizes (Bewick et al. 2004). The tables show the average SR and RR values with standard error, but the statistical analysis was performed on the median values of SR and RR. Statistical analysis and graphical presentation was done with MINITAB 17.3.1 and Microsoft Excel 2010 software (Version 14.0.4760.1).

\section{Literature review on RS sucrose concentration}

A set of 11 publications on droplet cryopreservation of potato shoot tips has been reviewed and classified by protocol steps, genotypes/taxa, recovery rates and assessment criteria (Table 1).

\section{Results and discussion}

A preliminary review of the literature on cryopreservation of different plant species using the droplet-vitrification method showed that most experiments traditionally used a RS containing a 1.2 M sucrose concentration for unloading ( $70 \%$ of the reviewed publications used a 1.2 M RS sucrose concentration). Specifically, for potato, 11 publications reported four different sucrose concentrations for unloading in RS: $0.09 \mathrm{M}, 0.8 \mathrm{M}, 1.0 \mathrm{M}$ and $1.2 \mathrm{M}$. As all steps of a protocol can influence directly or indirectly the efflux of CPAs (unloading efficiency) and influx of water (osmotic stress), a detailed comparison of the methodologies reported was warranted (Table 1).

The age of the in vitro plantlets used for shoot tips excision varied from 2 to 7 weeks and the shoot tip size from 1 to $4 \mathrm{~mm}$. The intracellular water and ion content of shoot tips coming from older and younger plants may vary, consequently the intrinsic osmotic potential could be different, affecting the influx of water during unloading. In larger shoot tips (2-4 mm) unloaded CPAs and incoming water may need to move longer distances than in shorter shoot tips (1-2 mm). Further, cold hardening and pre-culture steps can significantly influence the intracellular water content of potato shoot tips as shown by Folgado et al. (2015). Pre-culture and cold-hardening times, as well as pre-culture sucrose concentrations (0.09-0.7 M), varied considerably between the reviewed protocols. As the potato genotypes were treated with different cryoprotectant agents, it makes sense that RS sucrose concentrations may also need to be varied. For example, unloading of DMSO may, or may not, require different RS sucrose concentrations and unloading times than PVS2 (Kryszczu et al. 2006; Schäfer-Menuhr et al. 1997; Kaczmarczyk et al. 2008). Additionally, the conditions of recovery (light vs. darkness) and the composition and sucrose concentration of the recovery media may cause different post-unloading stresses as the differential of sucrose concentrations between RS and recovery media may affect water influx during recovery. Finally, different response variables were used for the assessment in the reviewed publications. Some authors reported complete plant recovery (Schäfer-Menuhr et al. 1997; Vollmer et al. 2017), while others reported only survival (swollen shoot tips of $>3 \mathrm{~mm}$ ) or shoot formation (Kim et al. 2006; Panta et al. 2015).

On a smaller scale, the effect of RS sucrose concentration and unloading times was previously assessed by Kim et al. (2006) where an assessment of a combination of unloading times $(10,30,60 \mathrm{~min})$ and sucrose concentrations $(0.3 \mathrm{M}, 0.8 \mathrm{M}$, and $1.2 \mathrm{M})$ was done using two potato varieties, var. 'Dejima' (S. tuberosum) and 'STN13' ( $S$. 


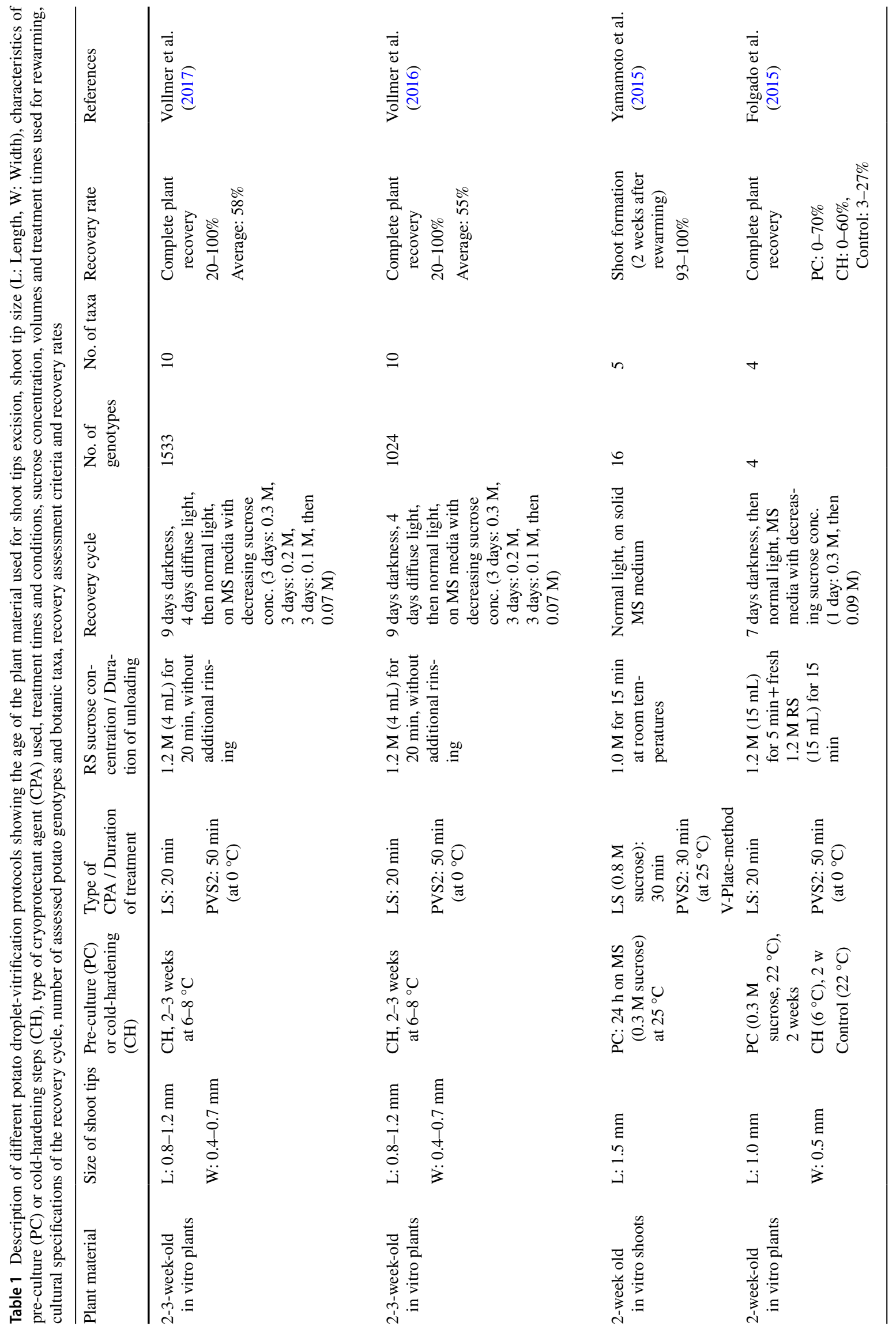




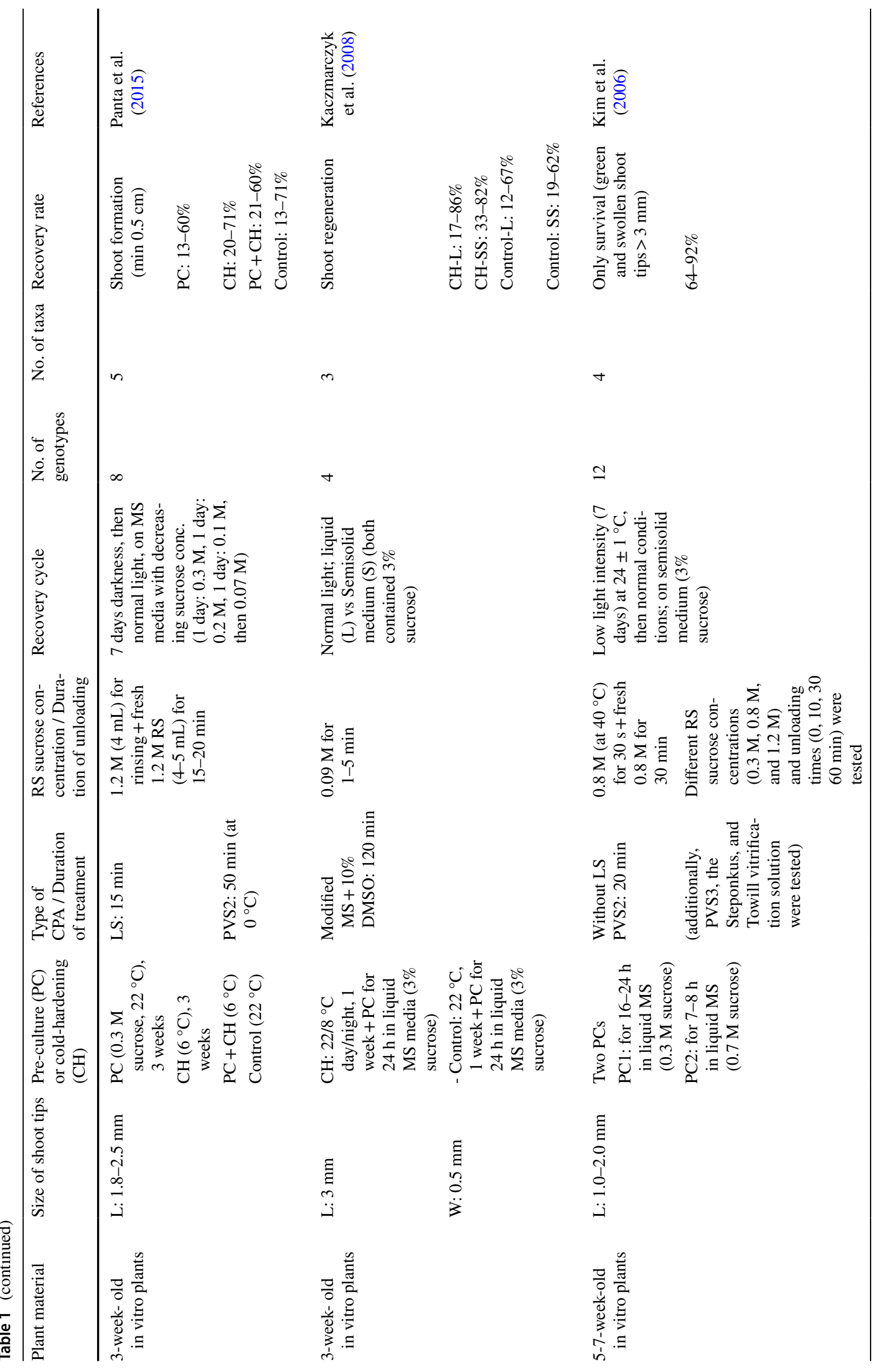




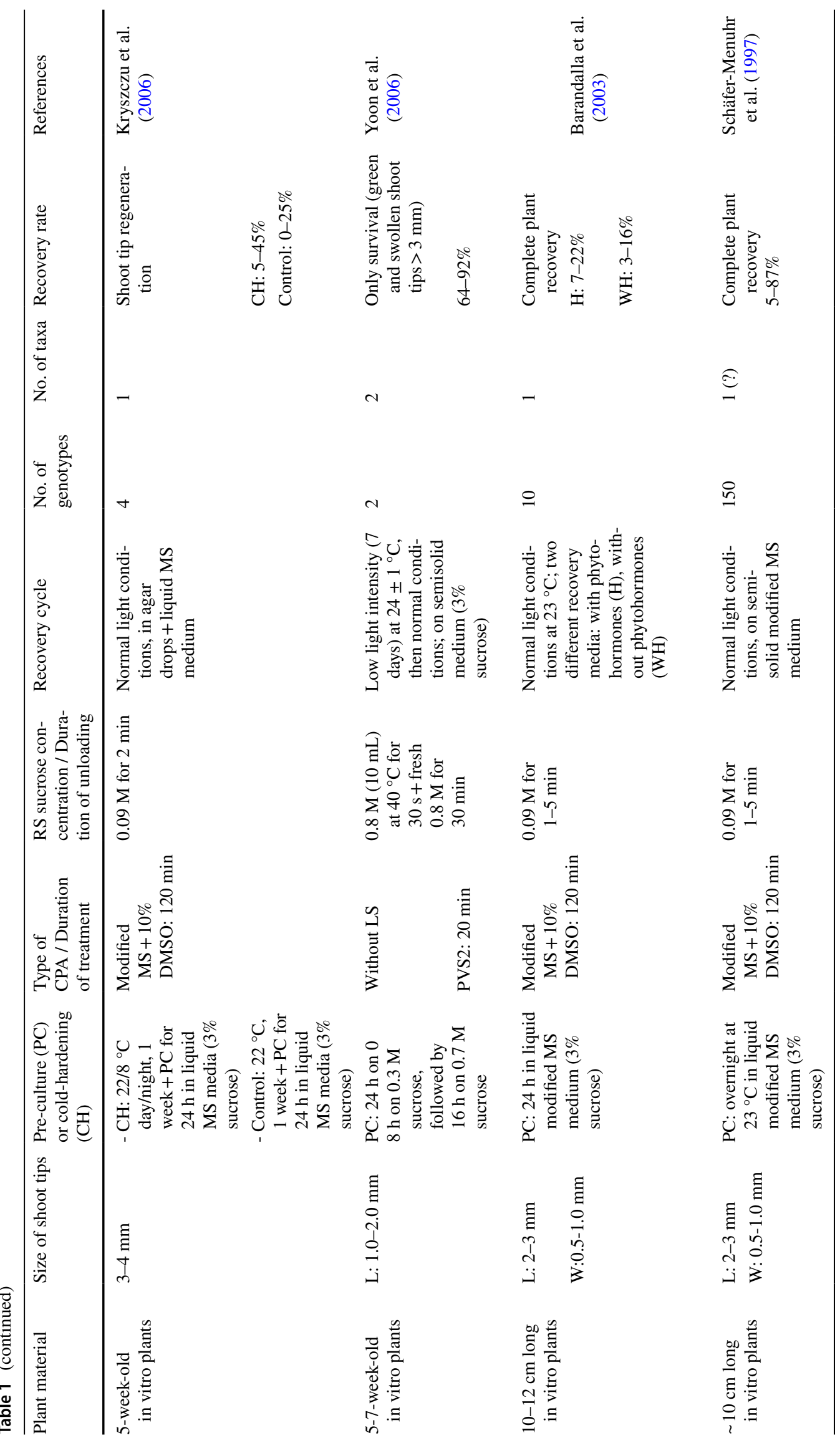


stenotomum subspecies stenotomum). The best unloading time and sucrose concentration based on these two varieties (30 min exposure to $0.8 \mathrm{M}$ sucrose RS) was tested on a set of 12 genotypes (belonging to 4 taxa). The reported survival rates ranged from 64 to $94 \%(+\mathrm{LN})$. Consequently, it may be possible that the unloading time in the present study of $20 \mathrm{~min}$ is not long enough to permit complete unloading of the cryoprotection solution (PVS2) or that transferring to a new RS solution (washing) is required.

Towill and Bonnart (2003) reported that cracking of external glass during warming did not result in a decrease of viability. Based on their results, it seems that the critical steps for successful and fast rewarming are not related to devitrification or thawing, but maybe influenced by a subsequent efflux of CPAs and influx of water into the shoot tips.

In the present study, the initial experiment with 16 potato accessions showed for the non-frozen samples $(-\mathrm{LN})$ a significantly higher median of RR with a RS sucrose concentration of $0.6 \mathrm{M}$ (means: $92.9 \%[-\mathrm{LN}], 86.3 \%[+\mathrm{LN}]$ ), compared to the $0.0 \mathrm{M}$ RS-sucrose concentration (means: $83.0 \%$ $[-\mathrm{LN}], 66.5 \%[+\mathrm{LN}])$. This small set of accessions (16) showed no significant differences between RR medians for RS in the remaining sucrose concentrations $0.3 \mathrm{M}, 0.6 \mathrm{M}$, $0.9 \mathrm{M}$, and the routinely utilized $1.2 \mathrm{M}$ (means: $81.1-86.8 \%$ ) (Table 2).

The data was evaluated to determine if genotype played a role in recovery rates. CIP 705458 (S. stenotomum subsp. goniocalyx) (mean RR: 93.8\%) showed a significantly higher median RR (+LN) than CIP 702353, CIP 704047, and CIP 706825 (S. stenotomum subsp. goniocalyx, S. $\times$ chaucha, and $S$. phureja, respectively) (means of RR: 64.9-69.8\%). Interestingly, when the data was pooled across all genotypes, cryopreserved shoot tips showed a low differential of $4.5 \%$ between the average SR (85.5\%) and RR (81.0\%) (Table 2). Three different patterns were observed among the genotypes which included: (I) six accessions (CIP 701611, CIP 702152, CIP 702439, CIP 704061, CIP 704087 and CIP 704043) had stable RRs (+ LN), independent of the RS sucrose concentration (0.0 M-1.2 M), (II) five accessions (CIP 702190, CIP 704052, CIP 705569, CIP 705458 and CIP 706250) showed lower RRs when sucrose was removed completely from RS (0.0 M), (III) five accessions (CIP 701561, CIP 702353, CIP 704541, CIP 704047 and CIP 706825) showed a higher $\mathrm{RR}$ with one of the intermediate RS sucrose concentrations (0.3 M, 0.6 M, or 0.9 M) (Fig. 1).

Somewhat surprising, complete removal of sucrose from RS $(0.0 \mathrm{M})$ resulted in an acceptable RR range of $46.7-86.7 \%$ (Fig. 1). This suggests genotype is an important factor that should be considered in response rates and further species and ploidy level do not seem to play a role in RR.

The results from this initial experiment, were expanded to a set of 85 diverse potato accessions, to determine if the RS sucrose concentration can be reduced to $0.3 \mathrm{M}, 0.6 \mathrm{M}$, or $0.9 \mathrm{M}$. RS sucrose concentrations of $0.3-0.9 \mathrm{M}$ resulted in significantly higher medians of the RR (means: 55.2-61.1\%) compared to the $0.0 \mathrm{M}$ and $1.2 \mathrm{M}$ RS-sucrose concentrations (RRs: 36.4-43.9\%) (Table 3), suggesting that RR is affected by the RS concentration employed.

No statistical differences were observed between the analyzed species/subspecies for the analysis of the decrease of the average recovery rate under osmotic stress conditions $(0.0 \mathrm{M}$ and $1.2 \mathrm{M}$ sucrose in $\mathrm{RS})$ in relation to the best bet RS sucrose concentration of $0.6 \mathrm{M}$. Although statistically non-significant, the $S$. phureja accessions showed a relative decrease of $16.6 \%$ [absolute RR: $36.7 \%$ ] in its average RR with a RS sucrose concentration of $1.2 \mathrm{M}$, compared to a relative decrease of 59.1\% [absolute RR: 18\%] when rewarmed in RS without sucrose $(0.0 \mathrm{M})$. S. xchaucha showed a nearly 3 -time higher relative decrease of $46.2 \%$ in RR for the same comparison (Table 3 , right column). In general, no clear correlation between species/subspecies and its response to increased osmotically stressful RS sucrose concentrations $(0.0 \mathrm{M}$ or $1.2 \mathrm{M})$ was observed.

Potato genotypes belonging to the same species/subspecies, show a variable response to the assessed RS sucrose
Table 2 Cumulative average survival and recovery rates of 16 potato accessions cryopreserved with the PVS2droplet method and rewarmed in rewarming solution (RS) with five different sucrose concentrations $(0.0 \mathrm{M}, 0.3 \mathrm{M}$, $0.6 \mathrm{M}, 0.9 \mathrm{M}$, and $1.2 \mathrm{M}$ )

\begin{tabular}{llllll}
\hline RS sucrose concentration & \multicolumn{2}{l}{$\mathrm{LN}$} & & \multicolumn{2}{l}{$\mathrm{LN}$} \\
\cline { 2 - 3 } \cline { 5 - 6 } & $\mathrm{SR}(\%) \pm \mathrm{SE}$ & $\mathrm{RR}(\%) \pm \mathrm{SE}$ & & $\mathrm{SR}(\%) \pm \mathrm{SE}$ & $\mathrm{RR}(\%) \pm \mathrm{SE}$ \\
\hline $0.0 \mathrm{M}$ & $88.3^{\mathrm{y}} \pm 3.0$ & $83.0^{\mathrm{y}} \pm 3.2$ & & $74.6^{\mathrm{b}} \pm 2.7$ & $66.5^{\mathrm{b}} \pm 3.3$ \\
$0.3 \mathrm{M}$ & $94.4^{\mathrm{x}} \pm 1.4$ & $90.0^{\mathrm{x}, \mathrm{y}} \pm 1.9$ & & $88.3^{\mathrm{a}} \pm 2.1$ & $84.2^{\mathrm{a}} \pm 2.1$ \\
$0.6 \mathrm{M}$ & $95.4^{\mathrm{x}} \pm 1.2$ & $92.9^{\mathrm{x}} \pm 1.6$ & & $89.1^{\mathrm{a}} \pm 1.7$ & $86.3^{\mathrm{a}} \pm 1.8$ \\
$0.9 \mathrm{M}$ & $93.9^{\mathrm{x}} \pm 1.2$ & $88.5^{\mathrm{x}, \mathrm{y}} \pm 1.9$ & & $90.7^{\mathrm{a}} \pm 1.6$ & $86.8^{\mathrm{a}} \pm 1.8$ \\
$1.2 \mathrm{M}$ (control) & $92.7^{\mathrm{x}, \mathrm{y}} \pm 1.4$ & $88.6^{\mathrm{x}, \mathrm{y}} \pm 1.7$ & & $84.7^{\mathrm{a}, \mathrm{b}} \pm 2.7$ & $81.1^{\mathrm{a}} \pm 2.8$ \\
Mean & $92.5 \pm 0.8$ & $88.2 \pm 0.8$ & & $85.5 \pm 1.3$ & $81.0 \pm 1.4$ \\
\hline
\end{tabular}

Different letters indicate significant differences between treatments based on the Kruskal-Wallis multiple comparison test for the median values $(\mathrm{p}<0.05)$

$S R$ survival rate; $R R$ recovery rate; $S E$ standard error; $-L N$ control treatment, without exposure to liquid nitrogen; $+L N$ shoot tips frozen in liquid nitrogen 


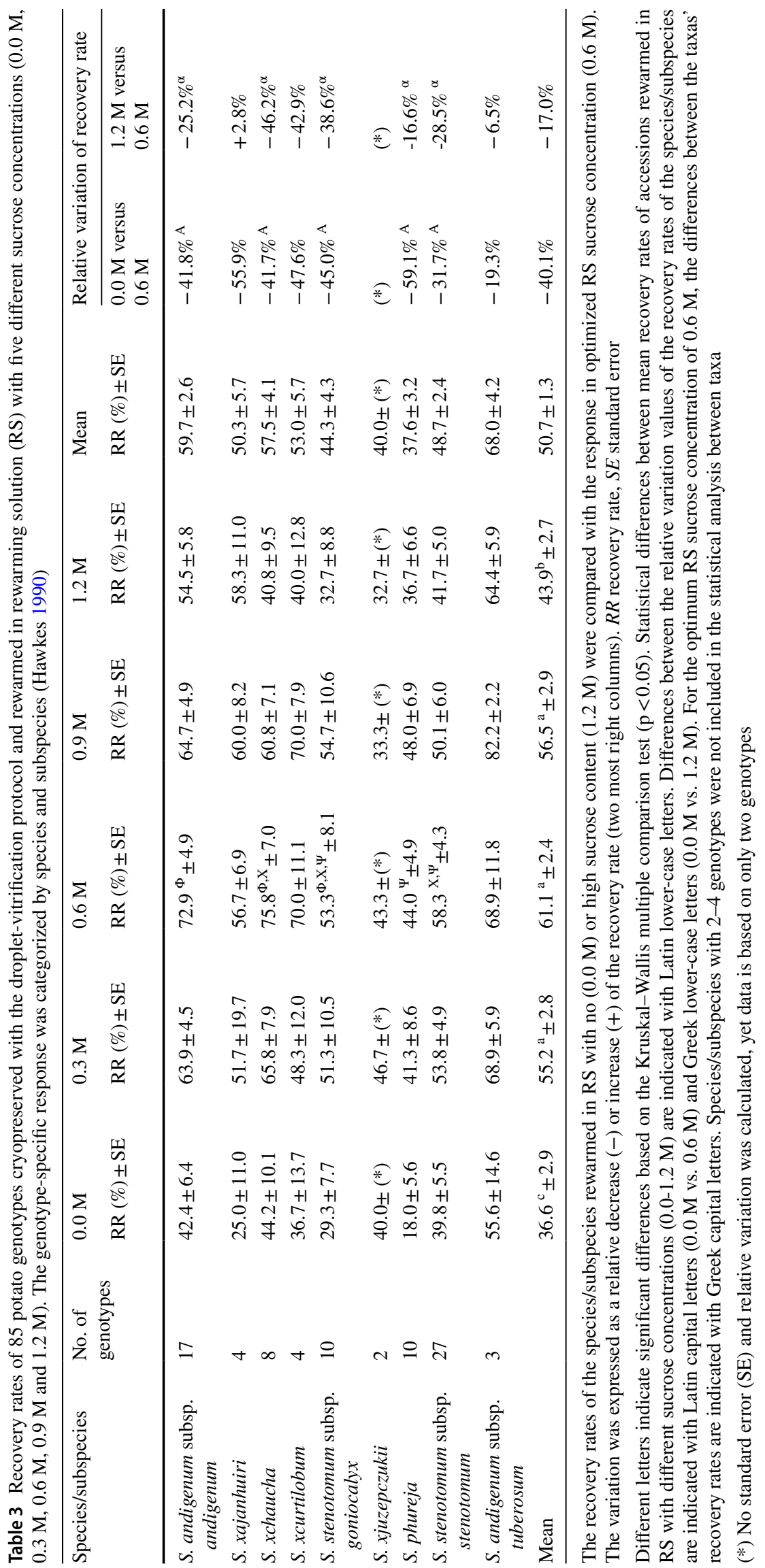




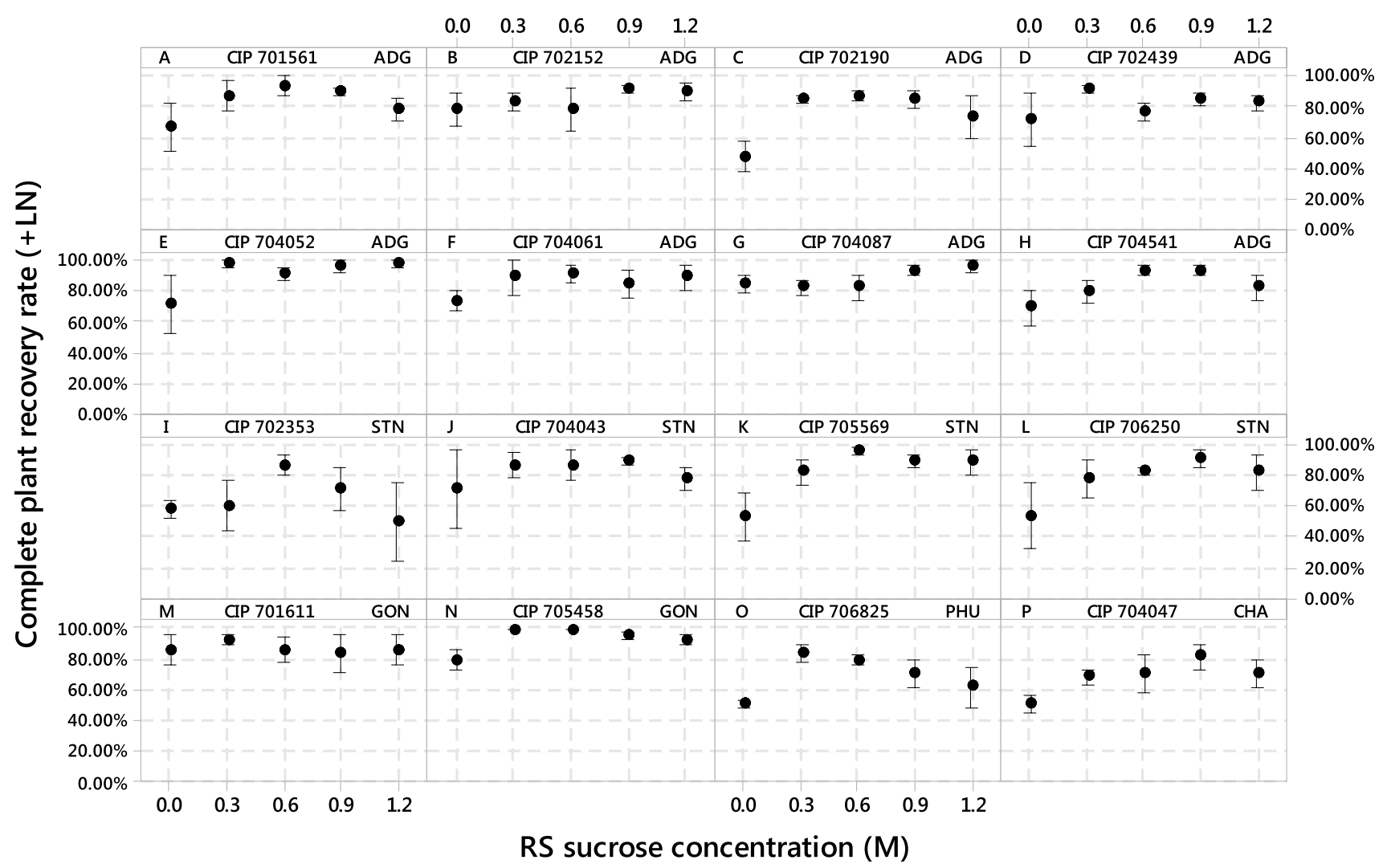

Fig. 1 Recovery rates $(+\mathrm{LN})$ of 16 potato accessions cryopreserved with the PVS2-droplet method and rewarmed in rewarming solution (RS) with five different sucrose concentrations $(0.0 \mathrm{M}, 0.3 \mathrm{M}, 0.6 \mathrm{M}$, $0.9 \mathrm{M}$ and $1.2 \mathrm{M}$ ). The standard error for each sucrose concentration

concentrations suggesting that it is likely genotype specific response. As an example, the accessions from S. tuberosum subsp. andigenum and $S$. xchaucha include both accessions that were sensitive (IDs 1, 2, 7, and 19) and tolerant to $0.0 \mathrm{M}$ (IDs 33, 34, 35, and 47) (Fig. 2). Similar results can be observed for other taxa. This led to the notion, that within the different potato taxa specific accessions are more tolerant to stresses during unloading with RS than others. It is assumed that water content of the cells before cryoprotection, influx and efflux capacity of cryoprotectant agents (CPAs) and water, and sensitivity to CPAs' toxicity and osmotic stress, vary greatly between genotypes of the same species/subspecies. Further, it might be possible that homologous groups of genes, that are present in specific accessions of different taxa, are strongly involved in the loading/unloading process of CPAs and/or control of influx/efflux of water (osmotic stress).

An analysis of the 85 screened accessions showed that 66 of the 85 accessions had the highest RR with a RS sucrose concentration of $0.6 \mathrm{M}$ (42 genotypes) or $0.9 \mathrm{M}$ (24 genotypes). In contrast, removal of sucrose from RS still resulted in a recovery rate of $30 \%$ or higher in 44 of 85 is indicated by bars. ADG: $S$. tuberosum subsp. andigenum, STN: $S$. stenotomum subsp. stenotomum, GON: S. stenotomum subsp. goniocalyx, CHA: S. × chaucha, PHU: S. phureja

accessions. The minimum acceptable recovery standard used at CIP is a RR of $30 \%$ (Vollmer et al. 2015). Six genotypes (CIP 703288, CIP 703314, CIP 703762, CIP 703952, CIP 706845, and CIP 707336) showed RRs of $80-100 \%$ when sucrose was completely removed from RS $(0.0 \mathrm{M})$; five of them belong to $S$. stenotomum subsp. stenotomum. Nine of the 85 genotypes showed a RR of $80 \%$ or higher for a RS sucrose concentration of $1.2 \mathrm{M}$ (CIP 701531, CIP 703520, CIP 703709, CIP 704501, CIP 704767, CIP 705352, CIP 705575, CIP 706213, and CIP 706764). These nine accessions belong to six taxa: S. tuberosum subsp. andigenum (4), S. xchaucha (1), S. stenotomum subsp. stenotomum (1), S. stenotomum subsp. goniocalyx (1), S. xajanhuiri (1), and S. phureja (1) (Fig. 2), again suggesting little correlation of taxa with RR in their response to sucrose levels in the RS.

Seventy-seven of 85 accessions were classified based on their response to the maximum and minimum RS sucrose concentrations $(0.0$ and $1.2 \mathrm{M})$, as well as, to their tolerance along the complete range of tested concentrations. The accessions were ordered and classified in four groups: (a) accessions sensitive to RS sucrose concentration of $0.0 \mathrm{M}$ (IDs 1-24), (b) accessions sensitive to a RS sucrose 


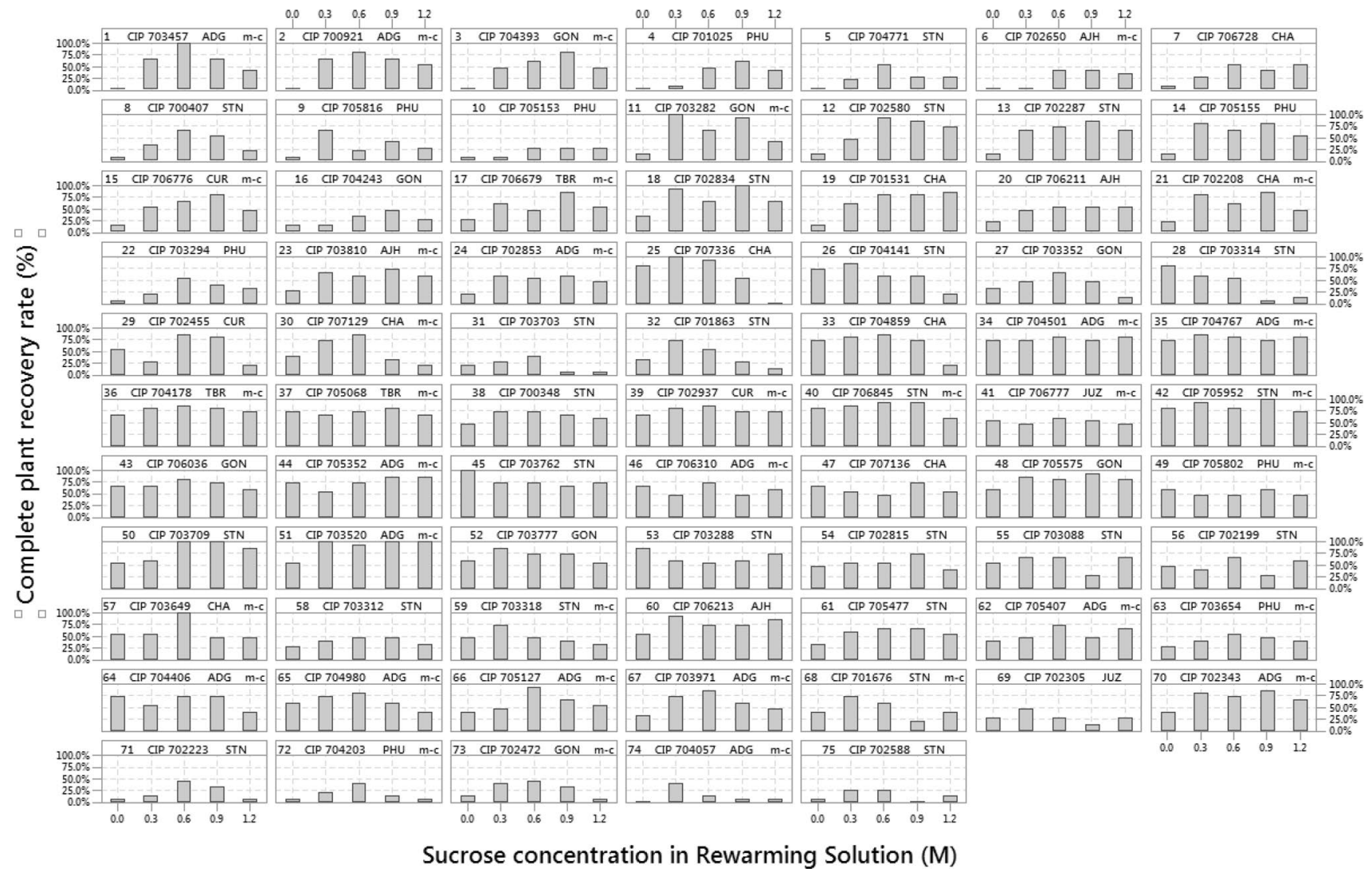

Fig. 2 Complete plant recovery rate (\%) of 75 of 85 assessed potato landraces (larger subset), cryopreserved with the PVS2-droplet vitrfication method and rewarmed in rewarming solution (RS) with five different sucrose concentrations $(0.0 \mathrm{M}, 0.3 \mathrm{M}, 0.6 \mathrm{M}, 0.9 \mathrm{M}$, and $1.2 \mathrm{M})$. The acessions were classified by it tolerance to RS sucrose concentration (see text). The header of each bargraph shows the following information: a ID (1-75), b accession number (CIP
XXXXXX), c species/subspecies identifier (ADG: S. tuberosum subsp. andigenum, TBR: $S$. tuberosum subsp. tuberosum, STN: $S$. stenotomum subsp. stenotomum, GON: S. stenotomum subsp. goniocalyx, PHU: S. phureja, AJH: S. xajanhuiri, CHA: S. xchaucha, CUR: S. curtilobum, JUZ: S. xjuzepczukii), d "m-c" if the accession forms part of CIP's mini-core collection

diverse set of 101 potato genotypes (16 acc. +85 acc.) over a wide range of RS sucrose concentrations $(0.0 \mathrm{M}, 0.3 \mathrm{M}, 0.6 \mathrm{M}$, $0.9 \mathrm{M}, 1.2 \mathrm{M})$. The results of this study showed, that complete removal of sucrose $(0.0 \mathrm{M})$, as well as, high RS sucrose concentration (1.2 M) significantly reduces the RR of cryopreserved potato accessions. It was noted that the use of $1.2 \mathrm{M}$ sucrose in RS is used as a standard concentration for many species and genotypes.

We postulate that cryopreserved potato clones show a highly genotype specific response to RS sucrose concentration during rewarming. Experimental results obtained with a limited number of accessions (16) should not be generalized for larger population sizes or collections (as is frequently done) because it does not always reflect the whole story as seen here. It would be interesting to perform similar experiments with species other than potato, to study the extent the most frequently reported RS sucrose concentration of $1.2 \mathrm{M}$ can be optimized. 


\section{Conclusions}

In an experiment with 16 clonal potato accessions, the use of RS sucrose concentrations of $0.6 \mathrm{M}$ and $0.9 \mathrm{M}$ showed on average $\sim 5 \%$ higher recovery rate, compared to the routinely used $1.2 \mathrm{M}$ sucrose concentration, suggesting that sucrose can be decreased in RS. A screening experiment with a set of 85 genetically diverse potato accessions confirmed this suggestion, and sucrose concentrations of $0.3-0.9 \mathrm{M}$ resulted in significantly higher recovery rates compared to the routinely used $1.2 \mathrm{M}$ sucrose concentration. The genotypic specific response to low $(0.0 \mathrm{M})$ and high RS sucrose concentrations (1.2 M) was variable within species/subspecies and ploidy levels. Interestingly, some genotypes showed high recovery rates of $\geq 80 \%$ even when sucrose was completely removed from the RS.

In general, the highest recovery was observed with a RS sucrose concentration of $0.6 \mathrm{M}$ ("best bet"), and therefore CIP's routine cryopreservation protocol for potato has been changed accordingly (from 1.2 M to 0.6 M RS sucrose concentration). We recommend being cautious in generalizing experimental cryopreservation results obtained with a limited number of accessions to larger collections.

Acknowledgements We express our sincere gratitude and thanks to the CGIAR Genebank Platform and the Global Crop Diversity Trust (GCDT) for funding this work. We also want to thank the following technical staff members for their continuous effort and commitment with CIP's potato cryobank: José Cárdenas, Anthony Alvarado, Maria Torres, Caren Senturón, Renso Cortez and Norca Pineda.

Author Contributions RV developed the experimental design, analyzed data and drafted the manuscript. RoVi and MC carried out the experiment. NA and DE supervised the project and contributed to the final version of the manuscript. All authors provided critical feedback and helped shape the research, analysis, and manuscript.

\section{Compliance with ethical standards}

Conflict of interest The authors declare that they have no conflict of interest.

Open Access This article is distributed under the terms of the Creative Commons Attribution 4.0 International License (http://creativeco mmons.org/licenses/by/4.0/), which permits unrestricted use, distribution, and reproduction in any medium, provided you give appropriate credit to the original author(s) and the source, provide a link to the Creative Commons license, and indicate if changes were made.

\section{References}

Barandalla L, Sánchez I, Ritter E, Ruiz de Galaretta JI (2003) Conservation of potato (Solanum tuberosum L.) cultivars by cryopreservation. Span J Agric Res 1(4):9-13. https://doi.org/10.5424/ sjar/2003014-43
Bewick V, Cheek L, Ball J (2004) Statistics review 10: further nonparametric methods. Crit Care 8(3):196-199. https://doi.org/10.1186/ $\operatorname{cc} 2857$

Farrant J, Walter CA, Lee H, McGann LE (1977) Use of two-step cooling procedures to examine factors influencing cell survival following freezing and thawing. Cryobiology 14:273-286

Folgado R, Panis B, Sergeant K, Renaut J, Swennen R, Hausman J-F (2015) Unravelling the effect of sucrose and cold pretreatment on cryopreservation of potato through sugar analysis and proteomics. Cryobiology 71:432-441. https://doi.org/10.1016/j.cryob iol.2015.09.006

Hawkes JG (1990) The potato: evolution, biodiversity and genetic resources. Belhaven Press, London

Hirai D, Sakai A (1999) Cryopreservation of in vitro-grown meristems of potato (Solanum tuberosum L.) by encapsulation-vitrification. Potato Res 42:153-160. https://doi.org/10.1007/BF02358405

Kaczmarczyk A, Shvachko N, Lupysheva Y, Hajirezaei M-R, Keller ERJ (2008) Influence of alternating temperature preculture on cryopreservation results for potato shoot tips. Plant Cell Rep 27:1551-1558. https://doi.org/10.1007/s00299-008-0574-1

Kim H-H, Yoon J-W, Park Y-E, Cho E-G, Sohn J-K, Kim T-S, Engelmann F (2006) Cryopreservation of potato cultivated varieties and wild species: critical factors in droplet vitrification. CryoLetters 27:223-234

Kryszczu A, Keller J, Grübe M, Zimnoch-Guzowska E (2006) Cryopreservation of potato (Solanum tuberosum L.) shoot tips using vitrification and droplet method. J Food Agric Environ 4(2):196-200

Murashige T, Skoog F (1962) A revised medium for rapid growth and bioassays with tobacco tissue cultures. Physiol Plant 15:473-497

Panta A, Panis B, Ynouye C, Swennen R, Roca W, Tay D, Ellis D (2015) Improved cryopreservation method for the long-term conservation of the world potato germplasm collection. Plant Cell Tissue Organ Cult 120:117-125. https://doi.org/10.1007/s1124 0-014-0585-2

Schäfer-Menuhr A, Schumacher H-M, Mix-Wagner G (1997) Longterm storage of old potato varieties by cryopreservation of shoottips in liquid nitrogen. Plant Genet Resour Newsletter 111:19-24

Towill LE, Bonnart R (2003) Cracking in a vitrification solution during cooling or warming does not affect growth of cryopreserved mint shoot tips. CryoLetters 24:341-346

Vollmer R, Villagaray R, Egúsquiza V, Espirilla J, García M, Torres A, Rojas E, Panta A, Barkley NA, Ellis D (2016) The potato cryobank at the International Potato Center (CIP): a model for long term conservation of clonal plant genetic resources collections of the future. CryoLetters 37(5):318-329

Vollmer R, Villagaray R, Cárdenas J, Castro M, Chávez O, Anglin NL, Ellis D (2017) A large-scale viability assessment of the potato cryobank at the International Potato Center (CIP). In Vitro Cell Dev Biol 53(4):309-317. https://doi.org/10.1007/s11627-017-9846-1

Yamamoto S-i, Wunna T, Rafique T, Valle Arizaga M, Fukui K, Cruz Gutierrez EJ, Castillo Martinez CR, Watanabe K, Niino T (2015) The aluminum cryo-plate increases efficiency of cryopreservation protocols for potato shoot tips. Am J Potato Res 92(2):250-257. https://doi.org/10.1007/s12230-014-9425-5

Yoon J-W, Kim H-H, Ko H-C, Hwang H-S, Hong E-S, Cho E-G, Engelmann F (2006) Cryopreservation of cultivated and wild potato varieties by droplet vitrification: effect of subculture of motherplants and of preculture of shoot tips. CryoLetters 27(4):211-222 